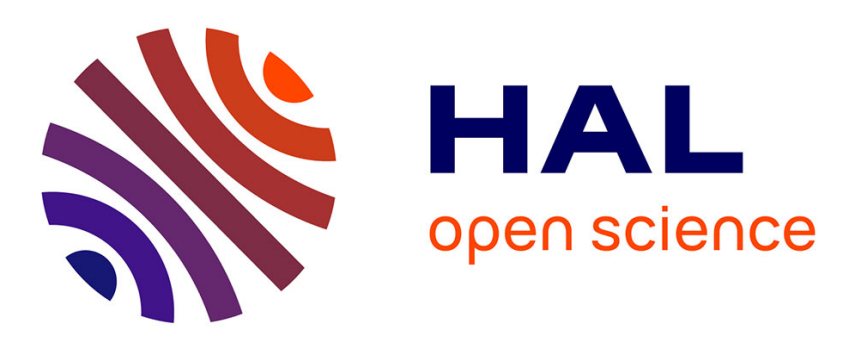

\title{
Jésus aux Îles Féroé, ou comment se réinvente la relation au divin
}

Christophe Pons

\section{To cite this version:}

Christophe Pons. Jésus aux Îles Féroé, ou comment se réinvente la relation au divin. Sophie Houdart et Olivier Thiery. Humains, non-humains. Comment repeupler les sciences sociales, La Découverte, pp.338-349, 2011, Humains, non-humains. Comment repeupler les sciences sociales. halshs-01144338

\section{HAL Id: halshs-01144338 \\ https://shs.hal.science/halshs-01144338}

Submitted on 21 Apr 2015

HAL is a multi-disciplinary open access archive for the deposit and dissemination of scientific research documents, whether they are published or not. The documents may come from teaching and research institutions in France or abroad, or from public or private research centers.
L'archive ouverte pluridisciplinaire HAL, est destinée au dépôt et à la diffusion de documents scientifiques de niveau recherche, publiés ou non, émanant des établissements d'enseignement et de recherche français ou étrangers, des laboratoires publics ou privés. 
Article paru dans Sophie Houdart et Olivier Thiery (ed.), Humains, non-humains. Comment repeupler les sciences sociales, La Découverte, 2011, Paris : 338-349.

JESUS AUX ÎLES FEROE, OU COMMENT SE REINVENTE LA RELATION AU DIVIN

\author{
Christophe Pons \\ Idemec-CNRS-AMU \\ cpons@mmsh.univ-aix.fr
}

Le nouveau Jésus débarqua dans les îles Féroé au début des années 1980. Dans cet archipel de 50.000 personnes (essentiellement originaires des îles), situé au nord-ouest de l'Ecosse, et sous protectorat danois, il chamboula les esprits et les coeurs. Ceux qui le rencontrèrent devinrent prosélytes d'une manière inédite, frappant aux portes, apostrophant leurs voisins dans la rue. Dans les fêtes de villages ils installaient des tribunes et donnaient témoignage de ce nouvel ami qui les avait transformés, qui les avait libérés, qui les aimait si fort. Au début, tout le monde trouvait cela étrange. Et puis, avec le temps, ces enthousiastes firent partie du décor. Partout dans les îles, à cause du nouveau Jésus, ils furent de plus en plus nombreux à quitter les communautés religieuses traditionnelles.

\title{
Jésus et les « enthousiastes »
}

Les anciens de ces communautés ne savaient trop qu'en penser. Ils ne pouvaient leur reprocher cette foi si enthousiaste mais ils constataient que, paradoxalement, elle les éloignait des congrégations familiales. Ceux qui avaient rencontré Jésus ne parlaient presque plus du Seigneur, Notre Père tout puissant. Ils n'avaient d'yeux et d'amour que pour son fils qu'ils présentaient comme leur ami. Ils s'adressaient à lui comme à un camarade ! Ils disaient le voir et l'entendre! Il y avait dans leur foi quelque chose d'impertinent, presque un manque de respect. Et pourtant, depuis qu'ils s'affirmaient libérés leurs vies étaient remarquables ; Ils ne fumaient ni ne buvaient plus, même en cachette, et leur temps libre était entièrement consacré à porter la bonne nouvelle. Pour la génération des anciens, ce comportement paraissait excessif, et inattendu. Car personne n'avait imaginé que les choses prendraient une telle tournure. Curieusement, tous avaient plutôt redouté que les îles ne cèdent au mouvement général de sécularisation qui semblait s'affirmer partout dans les pays d'Occident. Et ici, aux îles, on craignait que le libéralisme danois ne devienne la norme. Pour les vrais chrétiens, cela aurait été 
pire que tout: une identité chrétienne de façade, une foi peu profonde, une participation sans ferveur aux sacrements et offices de la liturgie calendaire. Bien sûr, une part de la société prit effectivement cette voie.

Aujourd'hui, plus de la moitié des féroïens appartient à l'Eglise Nationale par recensement, est baptisée à la naissance et reste loyale envers la coutume chrétienne qui fait l'histoire et l'identité de ces îles depuis plus de mille ans. Toutes les familles de l'archipel, même chrétiennes, ont en leur sein des grappes de séculiers qui ne viennent pas aux offices du dimanche et ne sont présents qu’à Noël et pour les grands sacrements : baptême, confirmation, mariage, funérailles. Le samedi soir, ils se rendent dans les pubs où ils fument et boivent ; ils ont des relations sexuelles hors mariage ; ils divorcent ; ils vont au Danemark pour avorter... Les chrétiens des communautés religieuses regrettent ces choix et les craignent pour leur moralité ; ils prient pour eux. Pour autant, les relations sont bonnes. Les uns et les autres se respectent mutuellement. Lorsqu'ils se retrouvent à l'occasion des grandes réunions lignagères, ils évitent simplement les sujets délicats et les objets de désaccord. A quoi bon les aborder? Ici, aux îles, on est si peu nombreux que l'on connaît l'art de vivre ensemble sans se fâcher. Pour le reste, chacun évolue dans son propre milieu.

La plupart des villages, depuis le $19^{\text {ème }}$ siècle, sont divisés en catégories majeures d'appartenance religieuse, délimitées par une frontière invisible. D’abord, ceux qu'on appelle les séculiers, qui se disent chrétiens parce qu'ils sont baptisés, mais qui ne vont à l'Eglise que trois fois dans l'année. Ensuite les luthériens qui appartiennent aux communautés dites Les Maisons de la Mission, et dans une proportion à peu près identique, les darbystes qui appartiennent aux communautés dites Les Assemblées des Frères. Ces deux groupes, luthériens et darbystes, sont les plus nombreux. Avec plus de soixante églises dans presque tous les villages, ils composent plus de $30 \%$ de la population totale. Mais curieusement, leurs relations ont toujours été très tendues, bien davantage qu'entre eux et les séculiers. C'est aussi que dans chaque famille chrétienne, de chacun des groupes, il y a toujours des séculiers tandis qu'il n'y a aucun luthérien chez les darbystes, et aucun darbyste chez les luthériens. De nos jours encore, ces deux moitiés demeurent parfaitement étanches l'une à l'autre. Enfin, parallèlement à quelques autres communautés de faible importance (Armée du Salut, Témoins de Jéhovah...), la nouvelle catégorie montante est celle des néo-pentecôtistes et des charismatiques. Les premières communautés pentecôtistes sont apparues dans les années 1920, mais elles sont aujourd'hui amalgamées aux néo- et charismatiques qui datent des années 1980. Ensemble, elles composent autour de 6\%. Mais ce sont elles qui rassemblent ceux qu'on nomme les enthousiastes, c'est-à-dire ces chrétiens exaltés qui, profondément bouleversés par leur rencontre avec Jésus, changent tout dans leur vie pour suivre ce nouvel allié.

Voilà donc le contexte global dans lequel le nouveau Jésus a débarqué dans les villages, frappant à la porte des cœurs séculiers, luthériens et darbystes. Chacun de ceux qui furent touchés par la grâce ne purent demeurer dans leur espace d'origine, leur relation singulière avec Jésus les entrainant trop loin sur des sentiers de traverse. C'est ainsi que Jésus a créé localement une petite révolution, multipliant les dissidences et brouillant les frontières invisibles qui, depuis fort longtemps, façonnaient le paysage religieux et social de ces îles du Nord.

\section{Le mysticisme comme voie d'individualisation des personnes}

De manière générale, les périodes historiques de crise mystique, où qu'elles se produisent et de quelque type qu'elles soient, sont toujours des moments privilégiés d'observation et d'analyse des existants non humains de la surnature (nous dirons ENHS), de leurs attributs, qualités, et de leurs modes d'action et d'intervention dans le temporel. Or, pour de nombreuses sociétés, notre époque contemporaine est l'une de ces périodes. Le nouveau 
Jésus, présenté ici à partir d'un regard indigène qui pointe précisément son action, connaît un succès étonnant bien au-delà du petit archipel des Féroé. Rarement en effet un ENHS a tissé autant d'alliances mystiques auprès d'individus issus de sociétés si diverses et si lointaines les unes des autres. Pour des millions d'individus à travers le monde, il est à présent un partenaire intime et personnalisé ; ceux-ci se "marient" avec lui, lui parlent, le voient, l'écoutent et le goûtent chaque jour de leur existence, en insistant sur le fait qu'il est vivant avec eux. Il s'agit donc bien d'une mystique, mais que nous dirons d'un " second type », au sens où il ne s'agit pas de la puissante abstraction en laquelle le sujet se laisse spirituellement absorber, mais d'une relation partenaire avec une entité anthropomorphisée, avec laquelle le sujet fait alliance, et en laquelle il trouve un ami, un camarade, parfois même un amant, mais qui en tous cas le révèle dans sa vie temporelle. L'affaire, on en conviendra, n'est pas nouvelle car c'est bien avec Jésus existant ambivalent puisqu'à la fois humain et divin - que le christianisme s'affirma dès le $2^{\text {ème }}$ siècle comme un monothéisme d'un genre différent. Cependant, cette forme relationnelle ne s'est vraiment mondialisée que tardivement, au cours du $20^{\text {eme }}$ siècle, avec la remarquable émergence d'églises néo-pentecôtistes et charismatiques à travers le monde.

A bien des égards, cette intimité développée entre l'individu et Jésus, qui éloigne d'une mystique classique en même temps qu'elle rapproche de formes relationnelles connues dans des systèmes religieux non monothéistes (chamanisme, possession, médiumnisme) ou dans des formes anciennes et coutumières de culte des saints (Brown 1984), invite à considérer avec attention l'interaction qui s'opère entre l'ENHS et le sujet. Cependant, longtemps l'anthropologie a écarté la possibilité de telles interactions, craignant de prêter aux ENHS une intentionnalité. Ceux-ci sont dès lors demeurés dans un statut de représentations ou croyances, inertes artefacts auxquels seuls les humains accorderaient qualités et actions. Mais cette modalité d'appréhension, fort raisonnable, a paradoxalement quelque chose d'anti-sociologique au sens où elle s'affranchit de la leçon durkheimienne sur les lois sociales : la société, les collectifs, sont mus par des lois qui ont leur propre autonomie indépendamment des individus qui la constituent. Faire de la sociologie, c'est accepter la possibilité de ces lois qui ne dépendent pas des acteurs mais qui sont la manière d'exister des collectifs. Et comme le rappelle Albert Piette, il n'en va guère autrement de tout existant non humain : "Dans les situations et les moments de la vie, nous le savons, les hommes rencontrent de nombreux êtres, humains ou non humains, vivants ou non vivants. Parmi ceux-ci, il y aurait donc les dieux, les collectifs et les animaux domestiques. Ce sont les « compagnons" des hommes" (2009: 140). Découvrir ces autres êtres relève de ce que Piette décrit comme une "phénoménographie de leurs modalités d'existence ", c'est-à-dire de leurs manières d'interagir, de se coordonner et de communiquer avec les hommes. Or, si cette "phénoménographie" est particulièrement essentielle aujourd'hui, c'est non seulement parce que les acteurs eux-mêmes n'ont de cesse de s'y référer, mais aussi parce que c'est dans ce face à face intime, personnel et privé avec l'existant Jésus, que cette crise contemporaine de mysticisme a effectivement débuté.

En somme, le nouveau Jésus n’est pas entré dans les sociétés par les portes des églises en place, mais par celles des cours individuels. Et à des échelles locales, comme celle de ces îles dont nous proposons l'examen, son débarquement a occasionné de profonds changements et créé la rupture avec des situations sociales qui étaient en place depuis fort longtemps. Quels sont dès lors les effets de son action, à la fois sur les psychologies individuelles et les collectifs ?

\section{Transgression et prestige social}

Si le débarquement du Nouveau Jésus fut ressenti dans les îles comme une rupture majeure, c'est parce que s'y sont associés des processus de transgression sociale et, par voie de conséquence, l'invention d'une forme nouvelle de prestige social. Ainsi, l'alliance avec le 
nouveau Jésus a permis aux individus de se comporter de manière inattendue et de bousculer des codes sociaux inscrits dans les mours insulaires depuis fort longtemps. Mais pour comprendre ce phénomène, il faut d'abord revenir sur la forme " traditionnelle " du prestige social vis-à-vis de laquelle, avec l'aide du nouveau Jésus, l'enthousiaste s'est démarqué.

La notion locale de good man (goður maður) renvoie aux dimensions religieuses des valeurs socialement estimées dans ces petites sociétés insulaires. Chez les darbystes comme chez les luthériens, dire de quelqu'un qu'il est un good man revient à reconnaître en lui sa haute moralité. Dans les faits, aucune procédure ne ratifie cette qualité. Il ne s'agit pas d'un statut mais d'une appréciation que la communauté, de manière implicite, porte sur ses individus au vu de leur conformation à un type exemplaire d'attitudes, de comportements et d'actes. Le profil type du good man est celui d'un père de famille, travailleur et entrepreneur, bon orateur et acteur dans son église. Ses prêches sont justes et fidèles aux écritures ; Il est sincère dans ses témoignages ${ }^{1}$. En somme, les qualités du good man sont celles du "berger " sachant guider son entourage, l'écouter et lui montrer la voie. Mais il ne doit pas avoir d'ambition pour lui-même, ni désirer occuper une position de dirigeant. Ceux qui réunissent ces qualités sont souvent sollicités pour faire partie des anciens, une distinction prestigieuse qui ne donne plus aujourd'hui d'indication d'âge. Pour d'autres, dont on dira aussi qu'ils sont des good men, les places et fonctions seront différentes : missions, activités diaconales, engagements divers... Mais ce qu'il importe de relever ici, c'est qu'à l'échelle de ces communautés religieuses acéphales, sans pastorat ni hiérarchie officielle ${ }^{2}$, où chacun est a priori sur un même plan d'égalité temporel ${ }^{3}$, la notion de good man ne renvoie pas à des qualités qui seraient héritées ou acquises au regard de l'ascendance, de l'éducation, du rang social ou du pouvoir financier; Elle ne témoigne pas non plus d'une position de domination ou d'autorité - ni temporelle ni spirituelle - mais renvoie l'individu à un prestige social qu'il aurait gagné au mérite, par approbation collective de sa moralité, et en fonction de sa conformation à un type idéal de mode d'être.

Il n'est pas anodin que cette notion de good man, qui ressort ici comme la forme idéalisée du prestige social, soit tout particulièrement prisée dans ces îles d'organisation communautariste, sans chefs héréditaires, où le mode de gouvernance opère de manière collégiale à l'échelle des segments qui composent l'unité villageoise. Et sous cette perspective, il est particulièrement tentant de rapprocher le good man féroïen du big man de certaines sociétés mélanésiennes puisque, d'une part, lui aussi " possède un pouvoir acquis grâce à ses propres mérites, donc non hérité et, en principe, non héritable. Ces mérites proviennent de la supériorité dont il fait preuve dans l'exercice de divers talents.»(Godelier, 1996 : 254). Et d'autre part, comme l'avait suggéré Marshall Sahlins, ce type de figure surgirait plutôt dans « des sociétés sans chefs héréditaires, acéphales, composées d'un certain nombre de groupes locaux, égaux sur le plan politique, administrant eux-mêmes leurs ressources matérielles et leur force de travail " (op.cit, 255), c'est-à-dire, en somme, structurellement proches de l'organisation féroïenne en villages segmentés par les communautés religieuses, modèle lui-même atypique dans le contexte européen". On sait que le modèle anthropologique décrit par Sahlins fut largement critiqué. Toutefois, l'analogie entre le big man et le good man nous permet de

\footnotetext{
${ }^{1}$ La notion n'est observée ici que pour les hommes. Un examen comparé du statut social et religieux des femmes mériterait cependant d'autres développements.

${ }^{2}$ Les communautés darbystes et luthériennes des îles Féroé n’ont pas de pasteurs mais sont acéphales avec un conseil d'ancien. Ceci les distinguent radicalement des églises pentecôtistes, néo- et charismatiques qui fonctionnent avec une autorité pastorale forte.

${ }^{3}$ Cette «égalité » de principe n'est vraie que sur un plan temporel et ne préjuge pas de ce pour quoi, sur un plan spirituel, l'individu est prédestiné. Là aussi, pour un examen plus serré de la notion et des transformations induites par l'arrivée d'une mystique par le nouveau Jésus, il faudrait repenser l'articulation entre les pôles de l'hérité, du mérité et du prédestiné.

${ }^{4}$ Atypique mais non pas aberrant. On retrouve en effet en partie les matrices de ce modèle dans les sociétés insulaires du Nord du Royaume Uni : Shetlands, Hébrides, etc.
} 
suggérer que certaines formes sociétales produisent certaines figures distinctives du prestige social. Et sous ce regard il semble en effet ressortir une probable continuité entre le type traditionnel d'organisation en unités segmentaires (les lignages du village), l'invention de formes communautaristes (congrégations religieuses d'appartenance) développées à partir de la fin du $19^{\text {eme }}$ siècle, et les qualités du good man au moyen desquelles les individus font localement «carrière en religion" (Pons 2009). Mais il ne s'agit pas de filer davantage l'analogie, d'autant qu'une caractéristique distinctive du good man par rapport au big man est qu'il n'est pas supposé prendre trop d'importance sur sa communauté, l'intérêt de celle-ci étant ce qui prime avant tout. Une différence essentielle serait ainsi que le big man cherche à accroître une autorité et une domination en amassant des richesses qu'il redistribue avec une générosité calculée (Godelier 1996), tandis que le good man est supposé demeurer un exempla de moralité sans chercher à asseoir son autorité ni à accroître un pouvoir. Or, voilà précisément ce que modifie l'alliance personnalisée avec le nouveau Jésus.

\section{Ce que Jésus fait aux individus...}

Avec Jésus, l'individu n'est plus contraint à rester en-deçà de l'intérêt de sa communauté religieuse d'appartenance. Car si Dieu, par l'intermédiaire de son fils, lui commande un projet de plus grande envergure, il n'a dès lors d'autre alternative que de se soumettre à cette volonté, ce qu'on ne pourra pas lui reprocher. L'effet induit par l'alliance mystique est dès lors tout à fait paradoxal puisque, d'un côté, le sujet est encore plus soumis à l'autorité surnaturelle mais, d'un autre côté, son ultra-soumission l'extraie de toute coercition sociale. D’une certaine manière, s'en remettre au Père en devenant "épouse " du fils revient à faire l'aveu de sa faiblesse, considérer que l'on est incapable de savoir ce qui est vraiment bon et, par conséquent, accepter de livrer son existence à un ENHS qui s'en servira à bon escient. Il y a dès lors, tout à fait explicitement, une conception adorciste de la possession de sa propre personne puisqu'on se donne à Dieu. Il nous possède tout à fait et dispose de nous. En échange, l'individu sait qu'il ne brûlera pas en enfer et que son âme sera sauvée pour l'éternité. Mais par-delà cet investissement sur son après vie, il gagne aussi quelques avantages terrestres. D’une part, en se donnant à Jésus, il se libère des entraves matérielles qui le contraignent ici-bas, il prend conscience de la valeur véritable des choses de ce monde, et affirme ainsi sa nature propre et profonde contre l'identité sociale qui lui était assignée de l'extérieur. Cette idée d'une libération du « soi original » est assez clairement estimée et formulée par les chrétiens qui renaissent dans leur alliance avec Jésus, et qui se disent libérés ou nés de nouveau - born again. D’une certaine manière, elle est même incluse dans la notion de salut; Non pas bien sûr d'un point de vue théologique mais pragmatique. Car les chrétiens qui renaissent deviennent enthousiastes non seulement du fait de la perspective de leur après vie, mais aussi par la métamorphose révélatrice qu'ils vivent dans leur existence ici-bas ; Ils sont enfin eux-mêmes, disent-ils ! Ceci indique que ce nouveau Jésus est bien le fruit d'une modernité, issu d'un processus de subjectivation par lequel l'individu se pense et se distingue du groupe. Mais d'autre part, ce " gain d'autonomie " lui donne aussi la possibilité d'agir dans ce monde d'une manière atypique, qui va le dégager des contraintes sociales qui l'enfermait dans le statut de good man, mais tout en faisant de lui une personne "inattaquable", disposant quasiment d'une sorte de statut d'impunité car il est “allié du fils"! En somme, il est intouchable et inatteignable car on ne pourra pas lui reprocher son alliance, ni d'être le serviteur de Dieu. Ainsi, dans les villages, l'une des premières formes majeures de dissidence au regard de la norme sociale fut de voir apparaitre des individus prosélytes qui, forts de leur alliance, frappaient aux portes, témoignaient sur la place publique de leur rencontre avec Jésus, et se rendaient même le samedi soir dans les pubs pour dissuader les séculiers de boire. Car jusqu'à présent, aux îles Féroé, on n'était prosélyte qu’à l'étranger, là 
où chaque communauté envoyait ses troupes de missionnaires ${ }^{5}$. Au village, en revanche, l'excès de zèle était mal vu : avec les séculiers, il est contraire au principe social de l'évitement du conflit, et avec les chrétiens, il ressort comme une atteinte aux églises concurrentes. En dehors de son lieu de culte, le seul prosélytisme qui soit localement admis est celui de l'exemplarité. C'est dire qu'on rejoint là encore les valeurs propres au good man puisqu'il s'agit, tout en restant discret, de susciter l'envie par son comportement. Rien à voir avec l'exaltation conquérante des époux du Christ qui pratiquent aux villages ce qu'on ne fait habituellement qu'en terre étrangère. Mais les enthousiastes franchissent encore les codes de la retenue sociale en faisant preuve d'une impudeur assumée, clamant qu'ils aiment les gens et qu'ils veulent leur bien. Là encore, si sur le fond les chrétiens n'ont rien à redire, la forme irrite. Et l'une des causes de l'agacement est aussi leur succès. Car on ne compte plus les luthériens et darbystes qui, grossis des rangs séculiers, se joignent aux réunions agitées des quelques églises enthousiastes des îles. C'est aussi que leur fréquentation est d'un genre nouveau ; Organisant des meetings autour de prédicateurs invités, souvent étrangers, les dévotions collectives attirent un public nombreux mais hétéroclite, moins attaché à son église que ne l'est habituellement le fidèle d'une communauté traditionnelle. On voit même apparaître des attitudes nomades par lesquelles les individus, curieux des diverses églises, vont assister aux cultes des unes et des autres. Cette forme inédite d'appartenance - ou de non appartenance - à une communauté est aussi le fait de la relation partenaire. Parce que Jésus est avec eux, les enthousiastes n'éprouvent plus la nécessité de s'enraciner dans une communauté de croyants. L'église, dès lors, n’est plus cette réalité d'un ordre supérieur qu'il s'agissait naguère de bâtir tous ensemble, comme un îlot du royaume de Dieu sur la terre. Cette vision, propre aux utopies ascétiques de tout le $19^{\text {ème }}$ siècle européen, avait fait de la communauté la seule voie de salut que le good man, par sa moralité, devait contribuer à construire. Désormais, l'église (avec le réseau, l'école, la mission et autres) est un des divers supports que l'enthousiaste emprunte afin d'œuvrer, en compagnie de Jésus, à un plan divin qui ne connaît plus de frontières. La vision de l'enthousiaste a donc changé d'horizon. Elle n'est plus la petite diaconie individuelle mais a gagné en autonomie et en ambition. Elle dépasse les frontières du village et même de l'archipel, chacun étant prêt à quitter son église pour nourrir ailleurs une mission plus vaste de nouvel apôtre.

Ainsi, par rapport au good man, si les termes de la moralité chrétienne demeurent identiques, l'enthousiaste se distingue par un libre arbitre assumé, et l'affirmation d'une autorité temporelle qu'il doit à sa soumission spirituelle. Car Jésus, en retour, gagne un fidèle qui lui est dévoué, qui s'engage à servir sa cause et à devenir prosélyte pour que d'autres humains se convertissent et s'engagent à leur tour dans cette bataille pour lui. La métaphore guerrière est amplement usitée. Jésus est un capitaine qui rassemble des troupes dans la perspective d'une victoire totale du royaume de Dieu sur toute la terre. Il s'adresse à chaque personne que Dieu a choisi, et leur confie la mission que celui-ci a décidé pour eux. Et c'est de cette façon que l'individu acquiert un nouveau pouvoir et prestige : en se livrant au public, en racontant son intimité avec Jésus. L’enjeu de sa distinction individuelle repose sur sa capacité à convertir autour de lui, c'est-à-dire à redistribuer la grâce qu'il a lui-même reçu de l'Esprit Saint (Coleman 2004). Par ce jeu de don (grâce donnée à l'individu par l'Esprit Saint dans l'alliance avec Jésus) et de contre don (l'évangélisation comme redistribution de cette grâce autour de soi), l'enthousiaste gagne un prestige social que l'on nomme charisme, et qui rappelle cette fois le prestige social acquis par le big man lors de l'incessant processus des dons et contre dons de richesses.

\footnotetext{
${ }^{5}$ Les îles Féroé sont le pays qui, par habitants, dispose du plus grand nombre de missionnaires à travers le monde, via divers réseaux internationaux tels que New Tribe Mission, Operation Mobilization, Mission Aviation Fellowship... Bien sûr, à leur retour aux villages, les missionnaires occupent généralement des positions importantes dans la hiérarchie des good men au sein de leur communauté religieuse. Les enthousiastes, quant à eux, empruntent plutôt d'autres réseaux de style plus charismatique : Youth With a Mission, Jesus Army, Jesus Revolution...
} 


\section{Quels effets sur la société ?}

Aux îles Féroé comme dans de nombreuses autres sociétés, la montée sensible du nombre des enthousiastes rappelle l'importance du champ religieux dans les processus de construction des identités individuelles, et dément le phénomène attendu d'un désenchantement. Ceci dit, pour les Féroé tout au moins, le principe d'intériorisation de la relation avec le divin n'est pas une nouveauté des trente dernières années. Dès la fin du $19^{\text {eme }}$ siècle, les conversions se produisirent sur ce modèle d'une expérience intime de la présence divine. Des individus, qui n'étaient nullement tenus pour dévots, se disaient soudainement touchés par la grâce, modifiaient leurs comportements, et rejoignaient - ou fondaient - les premières assemblées darbystes ou luthériennes. Leur statut social en était profondément affecté puisqu'ils occupaient des places nouvelles, au sein des hiérarchies de good men luthériens ou darbystes, qui émergeaient peu à peu à l'échelle des villages. La nouveauté de notre époque actuelle n'est donc ni dans la collusion entre le champ religieux et le prestige social, ni dans la modalité intériorisée de l'expérience divine par laquelle ce phénomène opère. Elle se situe en revanche, d'une part, dans l'autonomisation croissante des individus qui se désolidarisent à présent de leurs communautés religieuses. Non pas qu'ils soient sans communautés, mais plutôt que les parcours nomades deviennent normaux, de même que le fait de fréquenter plusieurs assemblées au cours d'une vie ; en comparaison, la norme chez leurs aînés good men était de rester affilié à la communauté sur un mode quasi organique. Et puis, d'autre part, la nouveauté incombe au fait que cette désaffiliation vis-à-vis de la communauté s'accompagne aussi d'une désaffiliation (relative) vis-à-vis de Dieu le Père, tandis que l'autonomisation s'accompagne d'une alliance personnelle (plus forte) avec Jésus le fils. En somme, sur le plan spirituel comme temporel, la valence de l'alliance dissidente supplante celle de la filiation policée. On voit donc ici à quel point les formes relationnelles dans les axes spirituels et temporels sont concomitantes l'une de l'autre et comment, pour notre propos, l'interaction entre l'ENHS Jésus et l'individu ne peut être exclue d'une compréhension non seulement des psychologies individuelles, mais aussi des changements d'échelle sociologique.

Notons, pour conclure, quelques-uns de ces effets majeurs sur le collectif féroïen. D’abord, les communautés traditionnelles ont été contraintes à une auto réévaluation. Elles ont réalisé qu'elles ne pouvaient rester repliées sur elles-mêmes mais devaient prendre acte des nouveautés introduites par le nouveau Jésus, notamment si elles désiraient contenir l'hémorragie des jeunes générations, de plus en plus attirées par les formes nouvelles de dévotion. Ce faisant, elles introduisent aussi, en leur sein, des débats (et des failles) autour, par exemple, des questions d'autorité (leadership), de "style» liturgique, ou de genre (les jeunes femmes charismatiques, également révélées par Jésus, ont de nouvelles aspirations). Tout ceci contraint donc les communautés à se poser la question de leur politique future, ce qui est bien évidemment source de dissensions et d'éventuelles scissions. La période actuelle est dès lors une époque de remaniement ou réorganisation du champ religieux dans sa globalité. Et ceci renforce une compétition entre communautés, car il s'agit non seulement de maintenir leurs présences mais surtout de l'étendre à de nouveaux territoires; de ce point de vue, les quartiers qui naissent à Tórshavn, la petite capitale, sont particulièrement signifiants de cette forte concurrence entre églises. En somme, le prosélytisme engagé par les enthousiastes a finalement influé sur l'attitude de toutes les communautés. Mais ce prosélytisme a aussi conduit à des formes collaboratives inédites entre ces communautés, en vue d'une action d'évangélisation plus large, destinée à la part séculière de la société féroïenne : nouvelle traduction de la bible, création d'une radio chrétienne et, bientôt, d'une chaîne de télévision. Ces actions, que l'on 
décrit localement comme oecuméniques ${ }^{6}$, créent la nouveauté d’un front chrétien qui s'unit et tend à faire bloc contre un "autre front ", moins clairement identifié, mais qui serait celui des séculiers. Au demeurant, au nom du principe de désamorçage des conflits, si profondément inscrit dans la mentalité de ces insulaires, rien n'a jamais été véritablement formulé en ces termes. Toutefois, depuis quelques années, l'opposition chrétiens versus séculiers commence à poindre épisodiquement, à l'occasion de débats qui, il y a peu, n'auraient jamais été abordés. Parmi ceux-ci, on trouve notamment la question du statut des femmes, le droit à l'avortement (interdit dans les îles, autorisé au Danemark), la reconnaissance de droits aux homosexuels ${ }^{7}$, les enseignements " créationniste " ou "darwiniste " à dispenser dans les écoles publiques. Sur tout cela, le nouveau Jésus impose ses opinions.

\section{Références}

Brown, P. 1984. Le culte des saints, Paris, Cerf.

Coleman, S. 2004. « The Charismatic Gift ", Journal of the Royal Anthropological Institute, 10 (3): 421-441.

Godelier, M. 1996. La production des grands hommes, (1982), Paris, Flammarion.

Piette, A. 2009. L'acte d'exister, Socrate Editions Promarex.

Pons, C. 2009. "The Problem with Islands: Comparing Mysticisms in Iceland and Faroe Islands ", Nordic Journal of Religion and Society, 22 (2): 43-61.

\footnotetext{
${ }^{6}$ Bien sûr, elles ne réunissent que les communautés chrétiennes dont les fondements théologiques sont à peu près acceptables par tous. Ainsi, par exemple, les témoins de Jéhovah en sont exclus.

${ }^{7}$ En 2002, un débat a violemment divisé la société sur cette question, et renforcé en même temps le parti chrétien au sein du gouvernement. L'enjeu était d'inscrire dans la constitution un nouvel article, stipulant qu'aucune personne ne doit faire l'objet de discrimination au regard de son orientation sexuelle.
} 